\section{Niveles séricos de alanino- aminotransferasa en población chilena: análisis de los resultados de la encuesta nacional de salud 2009-2010}

\author{
RODRIGO TEJOS ${ }^{1}$, OSLANDO PADILLA ${ }^{2}$, MARGARITA PIZARRO $^{1}$, \\ NANCY SOLÍS ${ }^{1}$, JUAN PABLO ARAB ${ }^{1}$, PAULA MARGOZZINI ${ }^{2}$, \\ JUAN FRANCISCO MIQUEL ${ }^{1}$, ARNOLDO RIQUELME ${ }^{1}$, MARCO ARRESE $^{1}$
}

\section{Serum levels of alanine aminotransferase in Chilean population: analysis of results of the national health survey 2009-2010}

Background: Determination of Alanine aminotransferase serum levels ([ALT] $]_{s}$ ) is a sensitive and reliable test for liver diseases. Aim: To report the prevalence of $a b$ normal [ALT] in Chilean population and to identify associated variables. Methods: We analyzed data from a random sub-sample of 2,794 adults surveyed during the second Chilean National Health Survey. Abnormal [ALT] were defined by using three different cut-off values (COV), two fixed COV (COV1: > 30 IU/L in men and $>19$ IU/L in women and COV2 pre-defined by the performing laboratory) and a COV adjusted by age, weight and sex (COV3 $>31 \mathrm{IU} / \mathrm{L}$ for women and $>44 \mathrm{IU} / \mathrm{L}$ and men $>42 I U / L$ and $>66 I U / L$ with a BMI > 23). Logistic regression analysis was performed to determine risk factors for elevated [ALT] Results: Mean $[A L T]_{\text {s }}$ values were $30.14 \mathrm{IU} / \mathrm{L}$ in men and $22.03 \mathrm{IU} / \mathrm{L}$ in women. The observed prevalence of abnormal [ALT] $]_{s}$ defined by different COV were 38\%, 11.5\%, and $8.1 \%$ for COV1, COV2 and COV3 respectively. Variables independently associated to abnormal [ALT]. in a multivariate analysis were the following: serum gamma-glutamyl-transpeptidase (OR:1.055 [95\% CI 1.033-1.078]) and body mass index (OR:1.13 [95\% CI 1.091.17]). Variables inversely associated with abnormal [ALT] $]_{s}$ (COV1) were male gender (OR:0.976 [95\% CI 0.96-0.99) and HDL-cholesterol (OR:0.979 [95\% CI 0.96-0.99]). Conclusions: Independently of the COV used, Chilean population exhibits a high prevalence of abnormal [ALT] $]_{s}$ which may reflect a significant burden of liver disease. Non-alcoholic fatty liver disease could be a major contributor to elevated [ALT]. considering the association of abnormal [ALT] $]_{s}$ and metabolic variables.

(Rev Med Chile 2013; 141: 909-916).

Key words: Alanine aminotransferase; Hepatitis; Hepatitis $C$ virus; Primary health care; Secondary prevention, fatty liver.

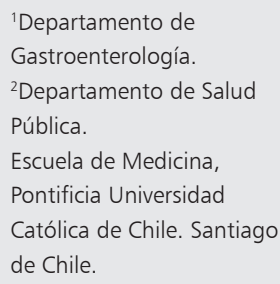

Financiamiento: La Encuesta Nacional de Salud (ENS) 2009 fue financiada por la Unidad de Estudios y Departamento de Epidemiología del Ministerio de Salud de Chile. El presente trabajo fue parcialmente financiado por la Comisión Nacional de Investigación Ciencia y Tecnología (CONICYT) de Chile (proyectos FONDECYT No 1110455 (M.A.) y No 1120652 (A.R.) y Anillo ACT79).

Los autores declararon no tener conflictos de intereses en este manuscrito.

Recibido el 2 de enero de 2013, aceptado el 7 de mayo de 2013.

Correspondencia a:
Dr. Marco Arrese J.
Departamento de
Gastroenterología. Escuela
de Medicina. Pontificia
Universidad Católica de
Chile. Marcoleta 367,
Casilla 114-D. Santiago,
Chile.
Teléfono: 56-2-3543820
E-mail: marrese@med.
puc.cl

I a alanina aminotransferasa (ALT) es una enzima que cataliza la transferencia de grupos amino para formar el metabolito oxaloacetato ${ }^{1}$. Se encuentra en abundancia en el citoplasma de los hepatocitos, donde su actividad es aproximadamente 3.000 veces mayor que en el suero ${ }^{2}$. En caso de daño o muerte hepatocelular, la liberación de ALT desde las células hepáticas incrementa sus niveles séricos. La ALT es considerada una enzima hígado-específica aunque también está presente en el riñón $y$, en cantidades mucho menores, en el miocardio y en el músculo esquelético ${ }^{3-5}$. Hay evi- 
dencias de que los niveles séricos elevados de ALT $\left([\mathrm{ALT}]_{\mathrm{s}}\right)$ se asocian a mayor mortalidad por causa hepática ${ }^{6}$ y podrían asociarse a mayor mortalidad por causa cardiovascular y diabetes ${ }^{7}$. En consecuencia, se ha sugerido que valores anormales de $[\mathrm{ALT}]_{\mathrm{s}}$ podrían ser un buen marcador de riesgo hepático y metabólico ${ }^{8,9}$. Entre las enfermedades hepáticas más comúnmente asociadas a valores anormales de $[\mathrm{ALT}]_{\mathrm{s}}$ se encuentran el hígado graso no alcohólico (HGNA), la infección crónica por virus hepatitis $\mathrm{B}$ o C, el consumo excesivo de alcohol y otras hepatopatías de menor prevalencia ${ }^{10}$.El HGNA es considerado la manifestación hepática de la obesidad y el síndrome metabólico (SM) $)^{11,12}$ y es la causa más común de elevación de enzimas hepáticas en la práctica clínica ${ }^{13}$ tanto como causa directa como contribuyente en presencia de otras etiologías ${ }^{14}$.

La medición de $[\mathrm{ALT}]_{\mathrm{s}}$ constituye una herramienta de tamizaje ("screening") de bajo costo, confiable y de amplia disponibilidad para detectar una enfermedad hepática asintomática ${ }^{15}$ y su determinación en la población chilena permitiría conocer la prevalencia poblacional de niveles anormales de esta enzima y generar información relevante para estimar la magnitud de la carga de enfermedad asociada a enfermedades hepáticas en nuestro país ${ }^{16}$. La aplicación de la segunda Encuesta Nacional de Salud (ENS) en Chile por el Ministerio de Salud (MINSAL) en el año 2009 permitió realizar determinaciones de $[\mathrm{ALT}]_{\mathrm{s}}$ en una muestra representativa de nuestra población. La presente comunicación reporta los resultados de la determinación de $[\mathrm{ALT}]_{\mathrm{s}}$ en población chilena y analiza las variables asociadas a niveles anormales de esta enzima. Es importante señalar que la definición de niveles de anormalidad de la [ALT] $]_{\mathrm{s}}$ es en la actualidad un tema en revisión ya que, dada la creciente incidencia de obesidad y sobrepeso tanto en Chile como en el mundo ${ }^{17}$, la distribución de los $[\mathrm{ALT}]_{\mathrm{s}}$ en la población aparentemente sana usada como referencia ha cambiado ${ }^{6}$. Por ello, se ha propuesto que los valores utilizados como referencia o puntos de corte de normalidad para la $[\mathrm{ALT}]_{\mathrm{s}}$ sean rebajados, ya que cuando se emplea una población de referencia con muy bajo riesgo de enfermedad hepática (definida por la negatividad de marcadores de infección por virus de la hepatitis B o C, bajo consumo de alcohol, ausencia de diabetes y normalidad de variables antropométricas [índice de masa corporal y circunferencia de la cintura]) los valores normales son menores a los usados convencionalmente $\mathrm{e}^{15,18,19}$. En el presente estudio se analizó la prevalencia de [ALT] anormales en población chilena empleando tres puntos de corte distintos, lo que permite estimar las implicancias de modificar el valor normal de esta enzima.

\section{Material y Métodos}

Este estudio es parte del análisis de los datos obtenidos de la segunda ENS (2009-2010). El grupo de población chilena estudiada en la ENS, corresponde a una muestra aleatoria de 5.412 personas, representativa de la población general, de ambos géneros, de residencia rural y urbana, mayor de 15 años, de todo el país. La metodología empleada para su selección se describe in extenso en el portal web del Ministerio de Salud de Chile ${ }^{20}$.

Los aspectos bioéticos de la ENS 2009-2010 siguieron las recomendaciones de estudios internacionales de esta naturaleza ${ }^{21-23}$. Además, el proyecto fue aprobado por el Comité de Ética de Investigación de la Escuela de Medicina de la Pontificia Universidad Católica de Chile. Los participantes en el estudio fueron examinados en sus hogares a través de al menos dos visitas de personal calificado para la aplicación de cuestionarios, el desarrollo de mediciones antropométricas y fisiológicas y la toma de muestras de sangre para las mediciones de laboratorio ${ }^{20}$.

Para el análisis de prevalencia de ALT anormal y factores asociados a esta condición, se utilizó una sub-muestra aleatoria de los sujetos seleccionados en la ENS 2009-2010. En este subgrupo $(\mathrm{n}=2.794)$ se determinaron los niveles séricos de ALT y gamma glutamil transpeptidasa (GGT). Dichas determinaciones, así como la medición de las concentraciones séricas de glucosa en ayunas, colesterol total, colesterol HDL y triglicéridos, fueron realizadas en un analizador automatizado Roche/Hitachi Modular P800 Chemistry Analyzer (Tokio, Japón).

La medición de la presión arterial se realizó con un aparato de presión automático Omron HEM 742 (Bannockburn, Illinois) promediando dos mediciones en la misma visita, separadas por 2 min, bajo condiciones de ayuno, horario matinal después de $5 \mathrm{~min}$ de reposo previo, vaciamiento vesical previo.

El SM, definido según criterios diagnósticos 
ATP III actualizados ${ }^{24}$, se diagnosticó cuando se encontraron presentes tres o más de las siguientes variables: circunferencia de cintura a la altura de la cadera $>102 \mathrm{~cm}$ en hombres y $>88 \mathrm{~cm}$ en mujeres, nivel de triglicéridos en suero $>150 \mathrm{mg} /$ $\mathrm{dL}$, concentración de colesterol HDL $<40 \mathrm{mg} /$ $\mathrm{dL}$ en hombres y $<50 \mathrm{mg} / \mathrm{dL}$ en mujeres, presión arterial $>130 / 85 \mathrm{mmHg}$ o el nivel de glucosa sérica $>100 \mathrm{mg} / \mathrm{dL}$.

En el caso de los niveles séricos de ALT, se consideraron anormales valores $>19 \mathrm{UI} / \mathrm{L}$ en mujeres $y>30 \mathrm{UI} / \mathrm{L}$ en hombres, de acuerdo a lo sugerido por Prati y cols. ${ }^{18}$. Adicionalmente se incluyeron otros puntos de corte en el análisis de los datos: a) los valores propuestos por Piton y cols. ${ }^{19}$ que categorizan según sexo e IMC y establecen como valores anormales $>31 \mathrm{UI} / \mathrm{L}$ para mujeres con $\mathrm{IMC} \leq 23 \mathrm{y}>44 \mathrm{UI} / \mathrm{L}$ para las que presentan IMC $>23$; para los hombres establecen como niveles de ALT anormales $>42 \mathrm{UI} / \mathrm{L}$ con $\mathrm{IMC} \leq 23$ y $>66$ $\mathrm{UI} / \mathrm{L}$ con IMC $>23$ y b) los valores pre-definidos por el Laboratorio Clínico de la Facultad de Medicina de la Pontificia Universidad Católica de Chile, que categoriza según sexo y edad, estableciendo como niveles anormales de ALT en mujeres de 18 años o más $>30$ UI/L y en menores de 18 años $>20 \mathrm{UI} / \mathrm{L}$. Para los hombres de 18 años o más establece como niveles anormales $>55 \mathrm{UI} / \mathrm{L}$ y $>$ $30 \mathrm{UI} / \mathrm{L}$ para menores de 18 años.

\section{Análisis estadístico}

El diseño del estudio corresponde a muestras complejas, donde cada individuo de la muestra es representativo de una cierta cantidad de sujetos de la población. Para la descripción de las variables continuas se utilizaron medias y error típico. Para la descripción de variables categóricas se utilizaron porcentajes. El análisis univariado para establecer asociación de las variables con la normalidad o no de ALT se realizó mediante regresión logística univariada. El análisis multivariado consistió en el ajuste de modelos de regresión logística que incluían las variables significativas en el análisis univariado, así como el sexo, la edad, IMC y el consumo promedio de alcohol diario durante la última semana (el cual considera consumo "0" para los sujetos que no tenían consumo en la última semana) e interacciones entre las variables, con selección de variables hacia atrás (backward). Los análisis se realizaron utilizando el módulo de muestras complejas del programa IBM SPSS
Statistics v17.0.0. Se calcularon los Odds-ratio (OR) y los intervalos de confianza (IC) 95\%. Las diferencias se consideraron significativas con valores de $\mathrm{p}<0,05$.

\section{Resultados}

En la ENS se entrevistaron 5.412 personas, entre las cuales se realizó la determinación de niveles séricos de ALT y GGT a 2.794 sujetos, los que constituyeron la submuestra analizada en este estudio. Las características generales de la submuestra poblacional se incluyen en la Tabla 1 . La edad media observada fue de 41,7 $\pm 0,55$ años. $51,1 \%$ de la muestra expandida correspondía a mujeres y $48,9 \%$ a hombres. El IMC promedio de la población fue de 27,31 $\pm 0,17$ lo que evidencia una elevada frecuencia de individuos con sobrepeso $^{25}$. El porcentaje de SM y de sospecha de diabetes mellitus en la sub-muestra expandida fue de $30,8 \%$ y $9,2 \%$ respectivamente.

Los valores promedio observados para los niveles séricos de ALT fueron 30,14 $\pm 0,99$ UI/L en hombres y 22,03 $\pm 0,63 \mathrm{UI} / \mathrm{L}$ en mujeres. Los valores promedio de GGT observados fueron $36,5 \pm 1,73 \mathrm{UI} / \mathrm{L}$ en hombres y $25,4 \pm 1,13 \mathrm{UI} / \mathrm{L}$ en mujeres.

Al estimar la prevalencia de niveles anormales de ALT en el suero en la población chilena, se observaron diferencias significativas dependiendo de cuál de los diferentes puntos de corte se utilizó para el análisis. Así, la prevalencia de $[\mathrm{ALT}]_{\mathrm{s}}$ anormales alcanzó a $38 \%$ si se utilizan los puntos de corte definidos por Prati y cols. ${ }^{18}$, fue de $11,5 \%$ si se emplean los valores de referencia pre-definidos por el laboratorio clínico donde se realizaron las determinaciones y fue de $8,1 \%$ si se sigue la recomendación de niveles normales de $[\text { ALT }]_{\mathrm{S}}$ propuesta por Piton y cols. ${ }^{19}$, que considera límites normales variables según el IMC (Tabla 2).

$\mathrm{El}$ análisis univariado de variables asociadas a niveles elevados de ALT, según Prati y cols. ${ }^{18}$ se muestra en la Tabla 3. Las variables que exhiben una asociación positiva con la presencia de niveles séricos anormales de ALT fueron: hipertensión arterial, IMC, diabetes mellitus, SM, glicemia, triglicéridos, colesterol total, colesterol-LDL, GGT y hemoglobina glicosilada. Los niveles del colesterol-HDL presentan una asociación inversa con la presencia de niveles anormales de ALT. La 
Tabla 1. Estadísticos expandidos para las características demográficas, antropométricas, clínicas y bioquímicas de la sub-muestra de la ENS 2009-2010 incluida en el análisis

\begin{tabular}{|c|c|}
\hline Sujetos ( $n, n$ expandido) & $2.794(7.561 .840)$ \\
\hline Sexo (hombres/mujeres)* & $1.151\left(48,9 \% \%^{a}\right) / 1.643\left(51,1 \%^{a}\right)$ \\
\hline Edad (años)** & $41,7 \pm 0,55^{b}$ \\
\hline \multicolumn{2}{|l|}{ NEDU } \\
\hline Alto* & $525\left(27,2 \%{ }^{a}\right)$ \\
\hline Medio* & $1.503\left(55,3 \% \%^{a}\right)$ \\
\hline Bajo* & $704(17,5 \% a)$ \\
\hline Urbano/Rural* & $2.423\left(89,9 \%{ }^{a}\right) / 371\left(10,1 \%^{a}\right)$ \\
\hline $\operatorname{IMC}\left(\mathrm{kg} / \mathrm{m}^{2}\right)^{* *}$ & $27,31 \pm 0,17^{b}$ \\
\hline Circunferencia de cintura $(\mathrm{cm})^{* *}$ & $94,7 \pm 1,83^{b}$ \\
\hline Índice cintura/cadera** & $0,97 \pm 0,019^{b}$ \\
\hline Consumo de alcohol (g/día)** & $60,22 \pm 6,09^{b}$ \\
\hline Sospecha hipertensión arterial* & $890\left(26,6 \%{ }^{a}\right)$ \\
\hline Sospecha diabetes mellitus* & $296\left(9,2 \%{ }^{a}\right)$ \\
\hline Síndrome metabólico (SM)* & $950(30,8 \% a)$ \\
\hline ALT Hombres/Mujeres (UI/L)** & $30,14 \pm 0,99 / 22,03 \pm 0,63^{b}$ \\
\hline GGT Hombres/Mujeres (UI/L)** & $36,5 \pm 1,73 / 25,4 \pm 1,13^{b}$ \\
\hline Glicemia $(\mathrm{mg} / \mathrm{dL})^{* *}$ & $93,47 \pm 0,89^{b}$ \\
\hline Colesterol total $(\mathrm{mg} / \mathrm{dL})^{* *}$ & $189,05 \pm 1,36^{b}$ \\
\hline Colesterol LDL (mg/dL)** & $113,43 \pm 1,14^{b}$ \\
\hline Colesterol HDL $(\mathrm{mg} / \mathrm{dL})^{* *}$ & $47,23 \pm 0,43^{b}$ \\
\hline Triglicéridos $(\mathrm{mg} / \mathrm{dL})^{* *}$ & $144,07 \pm 3,56^{b}$ \\
\hline
\end{tabular}

${ }^{*}$ n (\%), **promedio \pm error típico, aPorcentajes expandidos ${ }^{b}$ Media y Error Estándar expandidos † Laboratorio Clínico Central de la Facultad de Medicina de la Pontificia Universidad Católica de Chile. ENS: Encuesta nacional de salud, NEDU= nivel educacional, IMC = índice de masa corporal, ALT= alanino-aminotransferasa.

Tabla 2. Prevalencia de niveles anormales de alanino-aminotransferasa (ALT) en una sub-muestra de encuesta nacional de salud 2009-2010

\begin{tabular}{|c|c|c|c|c|}
\hline \multirow{2}{*}{$\begin{array}{l}\text { Punto de corte } \\
\text { (ref) }\end{array}$} & \multirow{2}{*}{$\begin{array}{l}\text { Valores de referencia de niveles } \\
\text { anormales de ALT }\end{array}$} & \multicolumn{3}{|c|}{ Prevalencia de valor anormal en población chilena } \\
\hline & & Total (\%) & Hombres (\%) & Mujeres (\%) \\
\hline Prati y cols. ${ }^{18}$ & $\begin{array}{l}\text { Mujeres > } 19 \mathrm{UI} / \mathrm{L} \\
\text { Hombres }>30 \mathrm{UI} / \mathrm{L}\end{array}$ & $1.071(38 \%)$ & 389 (33,6\%) & $682(37,6 \%)$ \\
\hline Piton y cols. ${ }^{19}$ & $\begin{array}{l}\text { Mujeres con IMC } \leq 23:>31 \mathrm{UI} / \mathrm{L} \\
\text { Hombres con IMC } \leq 23:>42 \mathrm{UI} / \mathrm{L} \\
\text { Mujeres con IMC }>23:>44 \mathrm{UI} / \mathrm{L} \\
\text { Hombres con IMC }>23:>66 \mathrm{UI} / \mathrm{L}\end{array}$ & $221 \quad(8,1 \%)$ & $83 \quad(7 \%)$ & $138 \quad(9,1 \%)$ \\
\hline $\begin{array}{l}\text { Pre-definido por } \\
\text { laboratorio* }\end{array}$ & $\begin{aligned} \text { 10-18 años: } & \text { Mujeres > } 20 \mathrm{UI} / \mathrm{L} \\
& \text { Hombres }>30 \mathrm{UI} / \mathrm{L} \\
>18 \text { años: } & \text { Mujeres }>30 \mathrm{UI} / \mathrm{L} \\
& \text { Hombres }>55 \mathrm{UI} / \mathrm{L}\end{aligned}$ & $386(11,5 \%)$ & $116(9,3 \%)$ & $270(13,7 \%)$ \\
\hline
\end{tabular}

\footnotetext{
* Laboratorio clínico de la Facultad de Medicina de la Pontificia Universidad Católica de Chile.
} 
Alanina-aminotransferasa en población chilena - R. Tejos et al

Tabla 3. Análisis univariado de variables asociadas a niveles de ALT según límite < 19 UI/L para mujeres y $<30 \mathrm{UI} / \mathrm{L}$ para hombres (Resultados expandidos)

\begin{tabular}{|c|c|c|c|c|c|}
\hline Variable & ALT normal & ALT anormal & Valor-p & OR & $95 \%$ IC \\
\hline Sexo/hombre (n/\%)* & $762(50,5 \%)$ & $389(46,1 \%)$ & 0,20 & 1,191 & $0,91-1,56$ \\
\hline Edad (años)** & $41,2 \pm 0,7$ & $42,6 \pm 0,8$ & 0,210 & 1,00 & $0,99-1,01$ \\
\hline Hipertensión arterial* & $530(23,5 \%)$ & $360(32,2 \%)$ & 0,003 & 1,55 & $1,16-2,06$ \\
\hline Diabetes mellitus* & $154(6,1 \%)$ & $142(14,8 \%)$ & $<0,001$ & 2,65 & $1,73-4,07$ \\
\hline Síndrome metabólico* & $459(22,0 \%)$ & $491(46,7 \%)$ & $<0,001$ & 3,11 & $2,30-4,20$ \\
\hline Consumo alcohol (g/día)** & $58,2 \pm 7,3$ & $63,5 \pm 10,6$ & 0,67 & 1,01 & $0,98-1,03^{+}$ \\
\hline Circunferencia de cintura $(\mathrm{cm})^{* *}$ & $93,0 \pm 2,7$ & $97,7 \pm 1,3$ & 0,3 & 1,01 & $0,99-1,03^{+}$ \\
\hline Índice cintura/cadera** & $0,98 \pm 0,3$ & $0,97 \pm 0,01$ & 0,57 & 0,95 & $0,79-1,14^{+}$ \\
\hline Glicemia $(\mathrm{mg} / \mathrm{dL})^{* *}$ & $89,8 \pm 0,5$ & $100,2 \pm 2,3$ & $<0,001$ & 1,17 & $1,1-1,25^{+}$ \\
\hline Colesterol total $(\mathrm{mg} / \mathrm{dL})^{\star *}$ & $183,6 \pm 1,7$ & $198,83 \pm 2,3$ & $<0,001$ & 1,09 & $1,06-1,13^{+}$ \\
\hline Colesterol LDL $(\mathrm{mg} / \mathrm{dL})^{* *}$ & $110,1 \pm 1,4$ & $119,4 \pm 1,9$ & $<0,001$ & 1,08 & $1,04-1,12^{+}$ \\
\hline Colesterol HDL (mg/dL)** & $48,7 \pm 0,6$ & $44,6 \pm 0,6$ & $<0,001$ & 0,75 & $0,67-0,84^{+}$ \\
\hline Triglicéridos $(\mathrm{mg} / \mathrm{dL})^{* *}$ & $125,6 \pm 3,6$ & $177,4 \pm 7,1$ & $<0,001$ & 1,05 & $1,03-1,06^{+}$ \\
\hline $\mathrm{GGT}^{* *}$ & $21,5 \pm 0,8$ & $47,8 \pm 2,4$ & $<0,001$ & 1,49 & $1,29-1,7^{+}$ \\
\hline $\mathrm{IMC} * *$ & $25,95 \pm 0,2$ & $29,7 \pm 0,3$ & $<0,001$ & 1,16 & $1,13-1,20$ \\
\hline Hemoglobina Glicosilada** & $6,0 \pm 0,1$ & $6,6 \pm 0,1$ & $<0,001$ & 1,34 & $1,15-1,57$ \\
\hline
\end{tabular}

${ }^{*} \mathrm{n}(\%),{ }^{* *}$ Promedio \pm e (error típico), +OR para cambio de 10 unidades.

edad, sexo, consumo de alcohol, circunferencia de cintura y el índice cintura/cadera no presentaron una relación estadísticamente significativa en el análisis univariado con los niveles de ALT.

Los resultados del análisis multivariado por regresión logística se muestran en la Tabla 4. Las variables independientemente asociadas a niveles anormales de ALT en el suero fueron: IMC y GGT, pero un aumento igual de GGT, en las mujeres representa un mayor riesgo de tener ALT elevado que el mismo aumento en hombres, lo que se traduce en que la interacción de sexo con GGT sea estadísticamente significativa en el modelo. El colesterol-HDL presenta una asociación inversa con la presencia de niveles anormales de ALT. La distribución por género de la alteración de los niveles séricos de ALT no varía al cambiar los puntos de corte utilizados para el análisis, manteniéndose constante la mayor proporción de mujeres con niveles de ALT alterados respecto de los hombres.
Tabla 4. Análisis multivariado por regresión logística de las variables asociadas a niveles elevados de ALT según límite < 19 UI/L para mujeres y $<30 \mathrm{UI} / \mathrm{L}$ para hombres

\begin{tabular}{|lll|}
\hline Variables & OR & IC 95\% para OR \\
Asociación positiva & & \\
IMC & 1,112 & $(1,077-1,147)$ \\
Sexo (masculino)*GGT & 1,030 & $(1,018-1,043)$ \\
Sexo (femenino)*GGT & 1,056 & $(1,037-1,075)$ \\
Asociación inversa & & \\
Colesterol-HDL & 0,979 & $(0,966-0,992)$ \\
\hline
\end{tabular}

Las variables e interacciones incluidas en el modelo fueron: sexo, nivel educacional, IMC, edad, consumo de alcohol (g/día), HTA, diabetes, síndrome metabólico, glicemia en ayunas, colesterol total, HDL, LDL, triglicéridos, GGT y sexo * consumo de alcohol (g/día), consumo de alcohol (g/día) * IMC, sexo * glicemia en ayunas, sexo * colesterol total, sexo * HDL, sexo * LDL, sexo * TGD y sexo * GGT, respectivamente. 


\section{Discusión}

Dado que la elevación de ALT es considerada un marcador fiable y sensible de enfermedad hepática, disponer de datos de prevalencia de niveles séricos anormales de esta enzima permite estimar la potencial carga de enfermedad asociada a enfermedades hepáticas en una población dada $\mathrm{da}^{6}$. La segunda ENS nos permitió determinar los [ALT], en una sub-muestra representativa y así estimar la prevalencia de su anormalidad en la población chilena. Para ello fue necesario definir los valores a usar como referencia de normalidad para los $[\mathrm{ALT}]_{\mathrm{s}}$. Los puntos de corte utilizados en la actualidad están en revisión porque se han generado en poblaciones de referencia donde personas potencialmente portadoras de enfermedades hepáticas asintomáticas fueron consideradas como sujetos sanos ${ }^{18}$. Ello se relaciona fundamentalmente con la inclusión en las poblaciones de referencia de individuos con sobrepeso potencialmente portadores de SM e HGNA ${ }^{11,12}$, y de pacientes portadores de infección por virus de la hepatitis C. En ese escenario, se decidió emplear tres diferentes definiciones de normalidad (Tabla 2). Así, según los valores normales de $[\mathrm{ALT}]_{\mathrm{s}}$ que se utilicen como referencia, se aprecian importantes diferencias en la prevalencia de $[\mathrm{ALT}]_{\mathrm{s}}$ alterados $[38 \%, 11,7 \%$ y $8,1 \%$ siguiendo las recomendaciones de Prati y cols. ${ }^{18}$, el laboratorio analizador y Piton y cols. ${ }^{19}$ respectivamente. La estimación de la prevalencia de $[\mathrm{ALT}]_{\mathrm{s}}$ anormales realizada en este estudio es muy similar a la reportada en estudios efectuados en Estados Unidos de Norteamérica en años recientes ${ }^{15,26}$. En particular, el estudio de Ruhl y Everhart ${ }^{15}$ definió que los valores de $[\mathrm{ALT}]_{\mathrm{s}}$ de $30 \mathrm{UI} / \mathrm{L}$ para hombres y $22 \mathrm{UI} / \mathrm{L}$ para mujeres, muy similares a los empleados como referencia en el presente trabajo, tenían el mayor poder discriminador de enfermedad hepática. Con dichos valores, los autores determinaron una prevalencia de $[\mathrm{ALT}]_{\mathrm{S}}$ anormales de $36 \%$ en la población norteamericana, similar al 38\% observado en población chilena. Al igual que en el presente trabajo, si se usan puntos de corte mayores, las cifras observadas se reducen hasta valores de $11 \%$. En todo caso, las prevalencias observadas tanto en Chile como en Norteamérica son de magnitud significativa en cualquiera de los escenarios, lo que tiene implicancias en términos de la necesidad de prevención y pesquisa de enfermedades hepáticas en nuestro país. Por otra parte, el análisis presentado hace evidentes las implicancias de la eventual adopción de las definiciones actualizadas de elevación de los [ALT] . Ello dado que el espectro de prevalencia varía hasta 3,5 veces definiendo proporciones notoriamente diferentes de adultos chilenos que requerirían una evaluación adicional de acuerdo a las recomendaciones internacionales ${ }^{27}$. Adoptar los límites normales de ALT sugeridos por Prati y cols. ${ }^{18}$ incrementaría, al aumentar la sensibilidad del examen de tamizaje, la detección temprana de enfermedades del hígado ${ }^{15}$, lo que debe balancearse con el impacto económico asociado al gasto determinado por la necesidad de estudios adicionales (marcadores de infección viral, marcadores de enfermedades autoinmunes, ultrasonografía, entre otros) eventualmente innecesarios en una proporción significativa de la población. Además, el eventual cambio de los valores de referencia de los [ALT] s podría tener implicancias para la definición de los criterios de diagnóstico, de control y de alta o terapia específica de infecciones por virus hepatotrópicos o enfermedades hepáticas autoinmunes.

Respecto de la etiología de la elevación de los [ALT] en este estudio, la asociación con parámetros de corte metabólico sugiere que el hígado graso, relacionado con la obesidad y resistencia insulínica, es una de las entidades más posiblemente relacionadas a dicha alteración de laboratorio. La prevalencia observada de infecciones por virus hepatotrópicos en la ENS 2009 fue muy baja $(<1 \%)^{24}$ por lo que dicha etiología no sería un contribuyente relevante. Por otra parte, el consumo de alcohol, a pesar de ser relevante en la población estudiada, no exhibe una asociación con la anormalidad de los $[\mathrm{ALT}]_{\mathrm{s}}$ en el análisis multivariado. Un factor que puede haber contribuido a esta observación es que la mayor parte de los chilenos bebe en forma intermitente y excesiva y que la alteración de [ALT] se asocia predominantemente al consumo regular de más de dos unidades por día ${ }^{28}$. El consumo de alcohol puede también contribuir directamente al desarrollo de alteraciones metabólicas, como la obesidad y la hipertrigliceridemia.

El presente estudio tiene limitaciones metodológicas inherentes a su naturaleza poblacional. Las elevadas frecuencias de obesidad y consumo de alcohol detectadas en la población y en la sub-muestra utilizada impidieron definir valores normales para la población chilena debido a que el 
número de individuos que cumplía criterios estrictos para ser considerados sanos era muy pequeño y, por tanto, no era representativo de la población general. El análisis de la literatura muestra que los valores sugeridos por Prati y cols. exhiben pocas variaciones al ser comparados con los obtenidos por otros autores en poblaciones diversas, incluyendo las de origen asiático ${ }^{29}$. En consecuencia, la prevalencia de ALT alterada observada en este trabajo puede ser considerada válidamente representativa. Otras limitaciones fueron no contar con mediciones seriadas de ALT, que podrían aumentar la sensibilidad y especificidad del examen en comparación con una medición aislada y no disponer de un examen ultrasonográfico del hígado para el diagnóstico de HGNA mide otros exámenes para descartar causas adicionales de enfermedad hepática (autoinmunidad, hemocromatosis, enfermedad de Wilson, etc.). Lo anterior, impide identificar con precisión las causas subyacentes a la presencia de ALT anormales. Sin embargo, la clara asociación de niveles anormales de ALT y variables de índole metabólico, incluyendo niveles anormales de GGT, que actualmente se consideran un buen marcador de resistencia insulínica e HGNA $^{30,31}$, permiten sospechar que la causa más probable de ALT anormal es el HGNA. Lo anterior es consistente con la elevada prevalencia de esta condición en nuestro país según estudios poblacionales recientes $^{32}$.

En resumen, el análisis de una submuestra de la ENS revela que una proporción significativa de la población (entre $8 \%$ y $38 \%$ ) exhibe niveles anormales de ALT lo que traduce que un porcentaje elevado de chilenos tiene riesgo de enfermedad hepática, posiblemente asociada a HGNA. El empleo de valores de referencia menores a los actualmente en uso tendría un impacto importante en términos del número de individuos que requerirían estudios adicionales.

\section{Referencias}

1. Price C. Biochemical assessment of liver function. In: Wright R, ed. Liver and biliary diseases-pathophysiology, diagnosis, management. London: W.B. Saunders, 1979: 381-416.

2. Wroblewski F. The clinical significance of transaminase activities of serum. Am J Med 1959; 27: 911-23.

3. Pratt DS. Liver Chemistry and Function Tests. In: Feldman M, Friedman LS, Brandt LJ, ed. Sleisenger \&
Fordtran's Gastrointestinal and Liver Disease, 9 th ed, Philadelphia, Pa: Saunders Elvevier 2010: 1227-36.

4. Siest G, Schiele F, Galteau MM, Panek E, Steinmetz J, Fagnani F, Gueguen R. Aspartate aminotransferase and alanine aminotransferase activities in plasma: statistical distributions, individual variations, and reference values. Clin Chem 1975; 21: 1077-87.

5. Cordoba J, O’Riordan K, Dupuis J, Borensztajin J, Blei AT. Diurnal variation of serum alanine transaminase activity in chronic liver disease. Hepatology 1998; 28 : 1724-5.

6. Kim WR, Flamm SL, Di Bisceglie AM, Bodenheimer HC. Serum activity of alanine aminotransferase (ALT) as an indicator of health and disease. Hepatology 2008; 47: 1363-70.

7. Yun KE, Shin CY, Yoon YS, Park HS. Elevated alanine aminotransferase levels predict mortality from cardiovascular disease and diabetes in Koreans. Atherosclerosis 2009; 205: 533-7.

8. Yildirim B, Ozugurlu F, Sahin S, Ozyurt H, Atis O, Akbas A, et al. Association between elevated aminotransferase levels and the metabolic syndrome in Northern Turkey. Ann Hepatol 2010; 9: 161-5.

9. Lee TH, Kim WR, Benson JT, Therneau TM, Melton LJ, 3rd. Serum aminotransferase activity and mortality risk in a United States community. Hepatology 2008; 47: 880-7.

10. Clark JM, Brancati FL, Diehl AM. The prevalence and etiology of elevated aminotransferase levels in the United States. Am J Gastroenterol 2003; 98: 960-7.

11. Goessling W, Massaro JM, Vasan RS, D’Agostino RB, Sr., Ellison RC, Fox CS. Aminotransferase levels and 20-year risk of metabolic syndrome, diabetes, and cardiovascular disease. Gastroenterology 2008; 135: 1935-44, 1944 e1.

12. Von Bernhardi R, Zanlungo S, Arrese M, Arteaga A, Rigotti A. [The metabolic syndrome: from an aggravating condition to a pathogenic risk factor for chronic diseases]. Rev Med Chile 2010; 138: 1012-9.

13. Méndez-Sánchez N, Arrese M, Zamora-Valdés D, Uribe $\mathrm{M}$. Current concepts in the pathogenesis of nonalcoholic fatty liver disease. Liver Int 2007; 27: 423-33.

14. Volzke H. Multicausality in fatty liver disease: is there a rationale to distinguish between alcoholic and nonalcoholic origin? World J Gastroenterol 2012; 18: 3492 501.

15. Ruhl CE, Everhart JE. Upper limits of normal for alanine aminotransferase activity in the United States population. Hepatology 2012; 55: 447-54.

16. Senadhi V. A paradigm shift in the outpatient approach to liver function tests. South Med J 2011; 104: 521-5.

17. Kac G, Velásquez-Meléndez G. [The nutritional transi- 
tion and the epidemiology of obesity in Latin America]. Cad Saude Publica 2003; 19 Suppl 1: S5, S4.

18. Prati D, Taioli E, Zanella A, Della Torre E, Butelli S, Del Vecchio E, et al. Updated definitions of healthy ranges for serum alanine aminotransferase levels. Ann Intern Med 2002; 137: 1-10.

19. Piton A, Poynard T, Imbert-Bismut F, Khalil L, Delattre J, Pelissier E, Sansonetti N, Opolon P. Factors associated with serum alanine transaminase activity in healthy subjects: consequences for the definition of normal values, for selection of blood donors, and for patients with chronic hepatitis C. MULTIVIRC Group. Hepatology 1998; 27: 1213-9.

20. Ministerio de Salud Chile. Segunda Encuesta Nacional de Salud, 2010: Capítulo III: Metodología.

21. Weir RF. Stored tissues Ethical, legal and Public Policy Implications. Iowa City: University of Iowa Press, 1998.

22. Lee LW, Griffith J, Zenick H, Hulka BS. Human tissue monitoring and specimen banking: opportunities for exposure assessment, risk assessment, and epidemiologic research. Environ Health Perspect 1995; 103 Suppl 3: 3-8.

23. Pappas G, Hyder AA. Exploring ethical considerations for the use of biological and physiological markers in population-based surveys in less developed countries. Global Health 2005; 1: 16.

24. Grundy SM, Cleeman JI, Daniels SR, Donato KA, Eckel RH, Franklin BA, et al. Diagnosis and management of the metabolic syndrome: an American Heart Association/National Heart, Lung, and Blood Institute Scientific
Statement. Circulation 2005; 112: 2735-52.

25. Ministerio de Salud Chile. Segunda Encuesta Nacional de Salud, 2010: Capítulo V: Resultados.

26. Ioannou GN, Boyko EJ, Lee SP. The prevalence and predictors of elevated serum aminotransferase activity in the United States in 1999-2002. Am J Gastroenterol 2006; 101: 76-82.

27. Green RM, Flamm S. AGA technical review on the evaluation of liver chemistry tests. Gastroenterology 2002; 123: $1367-84$.

28. Ruhl CE, Everhart JE. Joint effects of body weight and alcohol on elevated serum alanine aminotransferase in the United States population. Clin Gastroenterol Hepatol 2005; 3: 1260-8.

29. Park HN, Sinn DH, Gwak GY, Kim JE, Rhee SY, Eo SJ, et al. Upper normal threshold of serum alanine aminotransferase in identifying individuals at risk for chronic liver disease. Liver Int 2012; 32 (6): 937-44.

30. Thamer C, Tschritter O, Haap M, Shirkavand F, Machann J, Fritsche A, et al. Elevated serum GGT concentrations predict reduced insulin sensitivity and increased intrahepatic lipids. Horm Metab Res 2005; 37: 246-51.

31. Grundy SM. Gamma-glutamyl transferase: another biomarker for metabolic syndrome and cardiovascular risk. Arterioscler Thromb Vasc Biol 2007; 27: 4-7.

32. Riquelme A, Arrese M, Soza A, Morales A, Baudrand R, Pérez-Ayuso RM, et al. Non-alcoholic fatty liver disease and its association with obesity, insulin resistance and increased serum levels of C-reactive protein in Hispanics. Liver Int 2009; 29: 82-8. 